\title{
No hay tal revival. En torno a las disquisiciones filosóficas sobre la alienación en las postrimerias del siglo XX
}

\author{
Sofia Ache \\ Universidad de la República, Uruguay \\ sofia.ache@fic.edu.uy \\ https://orcid.org/0000-0002-0266-7576
}

\begin{abstract}
Resumen: El siguiente trabajo se propone dar argumentos para relativizar el extendido relato de que el concepto de alienación perdió atención teórica por parte de la filosofia durante la década de los ochenta del siglo pasado y que recién a partir de los noventa se produjo un revival en su estudio. La conclusión es que no hubo tal pérdida de atención sino que, además, las premisas asumidas en la discusión sobre la alienación desde los noventa hasta el presente fueron delineadas durante los ochenta. Esta conclusión tiene dos apoyos: el tipo de abordaje de la alienación por parte del marxismo analítico y las incursiones en el concepto llevadas a cabo en el marco de la sociología norteamericana. Con base en ello, se realiza una propuesta de sistematización de la historia del abordaje filosófico del concepto de alienación en dos tramos o etapas. Se trata de dos -y no de tres- tramos en el entendido de que, por lo que se sostuvo, la filosofia no dejó de hablar de alienación en los ochenta, aunque lo hiciera en mucha menor medida que antes (especificamente, durante los sesenta y los setenta, momentos considerados de boom en el tratamiento filosófico de la alienación).
\end{abstract}

Palabras clave: alienación; historia; filosofia; sociología; marxismo analítico

\begin{abstract}
There is no such Revival. Around the Philosophical Disquisitions on Alienation in the late Twentieth Century". The following paper proposes to give arguments to relativize the extensive account that the concept of alienation lost theoretical attention in the field of philosophy during the eighties of the last century, and that a revival of the study of the concept occurred in the nineties. The conclusion is that there was no such loss of attention, but that furthermore, the presumed premises in the discussion concerning alienation from the nineties up to the present were delineated during the eighties. This conclusion has two supporters: the type of approach regarding alienation carried out by analytical Marxism and the philosophical incursions into the concept in the context of North American sociology. Based on this, the text offers a systematization of the history of the philosophical study of the concept in two sections or stages. And it concerns itself with two -and not three- stages because, for what was argued, philosophy did not stop talking about alienation in the eighties, although it did so to a much lesser extent than before (specifically, during the sixties and seventies, considered the boom in the philosophical treatment of alienation).
\end{abstract}

Keywords: alienation; history; philosophy; sociology; western marxism. 


\section{Introducción}

En las discusiones de ciertas áreas de la filosofia desde hace algunos años ha comenzado a ganar terreno un concepto otrora muy popular: el concepto de alienación.

¿Qué es estar alienado y de qué formas esta cuestión sigue siendo tematizada por los filósofos contemporáneos? Sin pretender dedicar demasiado espacio a las dos arduas cuestiones -puesto que no constituyen exactamente el foco de este trabajo, de algún modo propedéutico con relación a las mismaspuede decirse que, si bien el uso del término "alienación" es rastreable hasta la antigüedad $^{1}$, el reconocimiento de una deficiencia personal y social que recibe tal nombre y que es objeto de elucidación filosófica tiene un origen propiamente moderno $^{2}$. Al igual que con otros tantos conceptos filosóficos, a partir del momento en que el tratamiento del concepto de alienación se extiende, diverge en un tejido amplio y diverso de sentidos que hoy conforman su rica constelación conceptual. De modo que, si bien no sería viable decir que hay una única definición de alienación, grosso modo e independientemente del planteamiento desde el cual se conceptualice, ser o estar alienado consiste en estar involucrado en una relación deficiente con uno mismo y con el mundo. Ello decir que, de algún modo, entre yo y algún aspecto de mí mismo o del mundo, unidos lógica, ontológica o normativamente, se ha producido una separación, un alejamiento, un extrañamiento, que se manifiesta en una pérdida de control de mí sobre ello y un sometimiento de mí mismo a ello. Es decir, el resultado es una pérdida de la libertad, lo cual contradice la concepción de vida libre que a partir de

\footnotetext{
1 El término griego originario designaba el cambio o la alteración en un sentido neutro, tanto como el deterioro y la adulteración, perversión y locura ( $c f$. DGE online: http://dge.cchs.csic.es /

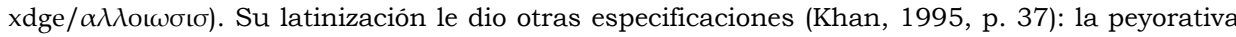
metáfora médico-psicológica que ya tenía, el sentido usual en el uso legal, vinculado a la transferencia de una propiedad, que la vuelve extraña (alien) a su antiguo dueño y la separación o alejamiento (el "éxtasis") que sufre una persona respecto de sí mismo, otros, su país o su dios. Algunos de estos usos persisten hoy, pero no resultan de interés a la filosofia política.

2 Los filósofos que hacen un racconto histórico del tratamiento filosófico del concepto de alienación señalan a Rousseau como el primer autor que da cuenta en sustancia del fenómeno. C f. vgr. Schacht, R., Alienation, Londres: George Allen \& Unwin LTD, 1971, pp. 10-13; Khan, N., Develop ment of the Concept and Theory of Alienation in Marx's Writings March 1843 to August 1844, Oslo: Solum, 1995, pp. 28-33; Schmitt, R., Alienation and Freedom, Cambridge: Westview Press, 2003, pp. 18-23; Fló, J., y M. Sambarino, Alcance y formas de la alienación, 2019, p. 14.
} 
la modernidad presuponemos tener o aspiramos a alcanzar como individuos. Estar alienado tiene implicaciones éticas, teórico-sociales y filosófico-sociales ${ }^{3}$. En el primer sentido, porque la alienación ataca a las condiciones de la autonomía personal, constituyendo una afrenta a la concepción de la vida buena de la persona. En el segundo sentido, debido a que la alienación es concebida como el efecto de un mal funcionamiento social que debe ser, por tanto, transformado. En el tercer sentido, por su parte, en virtud de que la alienación se entiende como una afección producida en el cruce de las relaciones yo-mundo, vinculada con las llamadas "patologias sociales"4, esto es, con deficiencias en las condiciones de autorrealización individual.

El entusiasmo de hoy por la alienación suele entenderse como un entusiasmo recobrado: luego de poco más de una década de silencio en torno al tópico, la filosofia ha vuelto a interesarse en el concepto de alienación, se dice. Elocuentes en este sentido resultan las expresiones de los primeros dos autores que trabajan la alienación a finales del siglo XX e inicios del presente siglo: Richard Schmitt y Richard Schacht. El primero comienza su libro diciendo que a pesar de que su trabajo está dirigido a estudiantes, "los filósofos profesionales se beneficiarán de leerlo porque el concepto de alienación ha sido poco discutido en la filosofía profesional de los últimos veinte o más años"5. Con respecto a las motivaciones del "rescate" del concepto que lleva a cabo en su obra The Future of Alienation, Schacht plantea que, si bien los ensayos que la componen formulan una perspectiva bastante crítica respecto a la literatura sobre alienación, "están destinados a funcionar como una especie de operación de rescate" 6 de la noción. Tal es así que, agrega, "[p]ara que la noción de alienación merezca un futuro, debe emplearse con mayor sofisticación y claridad de la que ha tenido... Este no es un sueño imposible -gracias, en parte precisamente, al declive de su antigua popularidad"7.

De los tratamientos del concepto que a partir del cambio de siglo se han llevado a cabo, el desarrollado por Rahel Jaeggi en su obra Entfremdung 8 es uno de los que más ha oxigenado la discusión. Junto a la obra de Jaeggi deben

3 Jaeggi, R., Alienation, XXII, Nueva York: Columbia University Press, 2016.

4 Un análisis del vínculo entre los conceptos de alienación y de patologías sociales lo lleva a cabo Pereira, G., El asedio a la imaginación, Granada: Comares, 2018, pp. 163-182.

5 Schmitt, R., Alienation and Freedom, Cambridge: Westview Press, 2003, p. vii. La traducción y el subrayado son míos.

6 Schacht, R., The Future of Alienation, The Future of Alienation, Urbana: University of Illinois Press, 1994, p. 15. La traducción y el subrayado son míos.

7 Ibid. El subrayado es mío.

8 Jaeggi, R., Alienation, Nueva York: Columbia University Press, 2016. 
mencionarse las más recientes incursiones de Jonathan Martineau ${ }^{9}$, Hartumut Rosa ${ }^{10}$, Franco Berardi ${ }^{11}$ y las no tan recientes de Richard Schmitt ${ }^{12}$ o Richard Schacht ${ }^{13}$ previamente citadas.

Sin embargo, no puede decirse sin incurrir en falsedad que este entusiasmo reciente sea un entusiasmo recobrado. Ello se debe a que, por una parte, la filosofia no dejó de hablar de alienación entre los años ochenta y los noventa -contrariamente a lo que estos autores afirman cuando se presentan a sí mismos como protagonistas de un revival del concepto, supuestamente sumido en el olvido durante ese tiempo-. Axel Honneth, al inicio del prólogo que realiza al libro de Rahel Jaeggi, contribuye a alimentar esta idea, al especificar la dificultad que el uso del término "alienación" comenzó a generar. Debido a los presupuestos esencialistas sobre los que descansaba el concepto tal y como había sido concebido por las tradiciones filosóficas que le dieron origen y continuidad -presupuestos inaceptables para los desarrollos filosóficos de décadas pasadas a ambos lados del Atlántico- la consecuencia fue que "la categoría de la alienación ha desaparecido del léxico filosófico" ${ }^{14}$.

Asimismo, tampoco es cierto que las motivaciones de la reapropiación del concepto de alienación que todos estos autores realizan sean inéditas hasta entonces, como se ejemplificará con el abordaje de Jon Elster. De todo lo anterior, se sigue que no puede decirse que el tratamiento de la alienación desde los noventa hasta ahora constituya una "nueva etapa" en la historia de la discusión del concepto por parte de la filosofia, y tampoco puede decirse que haya habido un quiebre o interrupción de la respectiva discusión en el intervalo comprendido entre los años ochenta y noventa.

Por lo tanto, este trabajo pretende dar elementos en apoyo a dos tesis. La primera tesis es que la filosofia no dejó de hablar de alienación o, como dice Honneth, que "la categoría de la alienación ha desaparecido del léxico filosófico"15. La segunda tesis es que la motivación en el tratamiento de la alienación no es tan original como se pretende. Para hacerlo, se realizará el

1989 Martineau, J., Time, Capitalism and Alienation. A Socio-Historical Inquiry into the Making of Modern Time, Boston: Brill, 2015.

${ }^{10}$ Rosa, H., Alienation and Acceleration. Towards a Critical Theory of Late-Modern Temporality, Malmö: NSU Press, 2010.

${ }^{11}$ Berardi, F., The Soul at Work. From Alienation to Autonomy, Los Ángeles: Semiotext(e), 2009.

${ }^{12}$ Schmitt, R., Alienation and Freedom.

${ }^{13}$ Schacht, R., The Future of Alienation.

${ }^{14}$ Honneth, A., "Foreword" en: Jaeggi, R., Alienation, Nueva York: Columbia University Press, 2016, p. vii.

${ }^{15}$ Ibid. 
recorrido que sigue: en primer lugar, se presentarán someramente las etapas de la historia de la discusión filosófica sobre la alienación y, posteriormente, se dará cuenta de dos contraejemplos a la visión de que la filosofía no habló de alienación en los ochenta y que a partir de los noventa lo hace con una motivación nueva.

1. La historia de la discusión filosófica sobre la alienación sin hacer demasiada historia. Un ensayo de sistematización

La historia de la reflexión filosófica sobre la alienación puede definirse de manera esquemática en dos momentos o etapas. La primera etapa se extiende desde la tematización rousseauniana de la alienación y pasa por Hegel, para bifurcarse hacia Karl Marx y Søren Kierkegaard.

Si bien suele decirse que en el año 1845 -en el que coescribe junto a Engels La ideología alemana- se produce un abandono expreso del término por parte de Marx, es necesario realizar algunas precisiones: si ese abandono por parte de Marx ocurrió o no corresponde al meollo de una discusión entre exégetas y seguidores del filósofo de Tréveris. Para algunos (Althusser y seguidores) los planteamientos acerca de la alienación corresponden a los textos de juventud de Marx y estos están en ruptura epistemológica con los tratamientos "maduros" del filósofo, aquellos, por tanto, no atendibles ${ }^{16}$. Para otros, en cambio, no hay ruptura sino continuidad entre los dichos de Marx sobre la alienación y los planteamientos posteriores tales como el fetichismo. Esa línea continuista podría hacer suyas las palabras de Henri Lefebvre: "La teoría económica del fetichismo retoma, eleva a un nivel superior, explicita la teoría filosófica de la alienación del individuo. Su actividad -el producto de su actividad-se presenta a él como otro, como su negación"17. Como expresión ex-ante en favor de la postura de Althusser cabe decir que la ortodoxia marxista nunca tematizó la alienación ni explícita ni implícitamente. Si bien podría admitirse que aún no se disponía del texto más importante de Marx para hacerlo (los Manuscritos Económicos y Filosóficos de Marx aparecen editados por primera vez en su lengua original en 1932), tampoco fueron suficientemente problematizadas cuestiones prácticas que tienen que ver con la alienación, vinculadas al lugar de los trabajadores en la revolución, qué tipo de transformación operaría en las conciencias en línea con la instauración del nuevo régimen y el rol del partido en ello; i.e., a grandes

${ }_{16}$ Althusser, L., La revolución teórica de Marx, México D.F.: Siglo XXI, 1964, p. 27.
Lefebvre, H., Le matérialisme dialectique, Paris: Quadrige, 1990, p.104.

ARETÉ Revista de Filosofía, v. XXXIII, 2, 2021 / e-ISSN 2223-3741 
rasgos, el problema de la racionalidad. Este tema es, sin dudas, discutible y complejo, y profundizar en él desviaría demasiado el propósito de este trabajo, pero una buena síntesis del panorama con relación a la situación del asunto de la alienación en la Unión Soviética la plantea Daniel Bell en "Dos vías desde Marx: los temas de la alienación y la explotación, y el control de los obreros en el pensamiento socialista" en El fin de las ideologías. Sobre el agotamiento de las ideas politicas en los años cincuenta (1960).

De acuerdo con Bell, la explicación de ese relegar remite a una especie de ingenuidad basada en "la creencia apocalíptica" de que "el día siguiente a la revolución" la racionalidad haría su aparición anunciada en la escena histórica y arreglaría toda la sociedad"18. Parecería ser que, producida la caída del capitalismo, la transformación de las conciencias sería instantánea, o, por usar un término de Rosa Luxemburgo, "espontánea". Como si la alienación (inherente, por lo tanto, al sistema capitalista únicamente) fuera a desaparecer con la caída del capitalismo y la instauración del nuevo orden. Ello lo constata Bell revisando algunas obras de Karl Kautsky, Lenin, León Trotski, en las que observa que el tratamiento sobre quién y cómo se llevaría adelante la transición a la nueva sociedad, e incluso el modo de organización y la planificación dentro de la misma son sumamente escasos ${ }^{19}$.

La otra precisión que debe realizarse tiene que ver con el uso del término por parte de tradiciones no marxistas. De dar por cierto que Marx abandona el término, no puede decirse sin incurrir en falsedad que no haya otro autor que lo utilice. Así, por ejemplo, dentro de la tradición existencialista cabe considerar los desarrollos kierkegaardianos en torno a la alienación, desperdigados en varias de sus obras por lo menos hasta el año 1849, año de publicación de su Sygdommen til Døden ${ }^{20}$. Desde otras tiendas, las disquisiciones sobre el Übermensch hechas por Nietzsche, fundamentalmente en su Asi habló Zarathustra de 1883, a veces son citadas como ejemplo de casos dentro de la discusión sobre la alienación (el superhombre se ubica, precisamente, en las antípodas del alienado ${ }^{21}$. Dado que tanto Kierkegaard y -en menor medidaNietzsche son considerados integrantes del grupo de filósofos que dieron cuenta

\footnotetext{
${ }^{18}$ Bell, D., "Dos vías desde Marx: los temas de la alienación y la explotación, y el control de los obreros en el pensamiento socialista”, en: Bell, D. (ed.), El fin de las ideologías. Sobre el agotamiento de las ideas politicas en los años cincuenta, Madrid: Centro de Publicaciones Ministerio de Trabajo y Seguridad Social, 1992, p. 409.

${ }^{19}$ Cf. ibid., pp. 411-415.

20 Traducido como La enfermedad mortal.

${ }^{21}$ Cf. Schmitt, R., Alienation and Freedom, pp. 32-37.
} 
de los efectos subjetivos de la alienación, eso pone en cuestión que el final de la primera etapa se coloque en 1845 y no en 1883. En razón de que la contribución de Nietzsche en la discusión fue marginal, tanto por su intención de aportar a la discusión misma como por la influencia que tuvo en otros pensadores que participaron en la segunda etapa de la discusión filosófica sobre la alienación, el final de la primera etapa sería un poco más tardío. Sin considerar a Nietzsche, este final es doble: para la vertiente no marxista, en 1849, para la vertiente marxista, en cambio, en 1845, si se acepta que Marx desiste de utilizar el término (abonando la lectura althusseriana de la obra de Marx). Si no se acepta la lectura althusseriana y sí la lectura continuista, y se asume con ello que Marx también habló de alienación, pero sin usar el término alienación en Das Kapital, entonces debe admitirse que la vía marxista se extiende por lo menos hasta 1867. A partir de este momento, por tanto, empezaria el primer silencio de la filosofia con relación a la alienación.

Hechas estas precisiones -que sirven para cuestionar la fecha en la que se fija el inicio del primer silencio- puede afirmarse que el primer silencio culmina en la línea marxista en 1923 con la publicación de "Reificación y la conciencia del proletariado" en el libro Historia y conciencia de clase de György Lukács, momento en el que se ubica, entonces, el inicio de la segunda etapa acerca de la alienación. En el caso de la línea no marxista, por su parte, el primer silencio acaba en 1927 con la publicación de Ser y Tiempo de Martin Heidegger. Por lo tanto, en 1923 y en 1927 se ubican los mojones del inicio de la segunda etapa de la discusión filosófica sobre la alienación.

Un aspecto muy relevante de la segunda etapa consiste en la puesta de moda del término, que lo condujo a perder sentido. Una explicación de ese boom podría vincularse a la publicación inglesa de los Manuscritos Económicos y Filosóficos de Marx en el año 195922. A partir de la publicación inglesa, los estudios acerca de Marx sufrieron un estallido que determinó la proliferación indiscriminada del uso del término por parte de diversos autores ${ }^{23}$, generando una suerte de "panalienación" ${ }^{24}$ en los abordajes sociales y filosóficos en los

22 Este dato lo aporta Fromm, en una nota al pie en su clásica obra de 1961. Cf. Fromm, E., Marx y su concepto del hombre, México D.F.: Fondo de Cultura Económica, 1964, p. 17.

23 Sería una hazaña dar cuenta de todos ellos. No es objetivo de este trabajo hacerlo, sino solo tomar como cierto el aserto de que realmente el estallido fue tal. El lector desconfiado puede comprobarlo refinando la búsqueda por fecha del término "alienation" en cualquier metabuscador.

${ }^{24}$ Musto se refiere a este momento como la "época de la alienación tout court" (Musto, M. (ed.), De regreso a Marx: nuevas lecturas y vigencias en el mundo actual, Buenos Aires: Editorial Octubre, 2015). Esa fascinación que produjo la alienación en esa época la hizo integrar el imaginario de la 
sesenta y setenta. En estos abordajes, el fenómeno de la alienación es tomado como un hecho que fagocita cualquier posibilidad de escape, invadiendo asimismo cada aspecto de la vida analizada. El caso de Erich Fromm es significativo en ese sentido ${ }^{25}$, así como el de Herbert Marcuse en One-Dimensional Man. Entre las obras del boom, Musto incluye las obras: La sociedad del espectáculo (1967) de Guy Debord y La sociedad del consumo (1970) ${ }^{26}$ de Jean Baudrillard. Asimismo, el fenómeno de la alienación se encuentra plasmado en los análisis más representativos de la primera generación de la Teoría Crítica como Dialéctica del iluminismo de Adorno y Horkheimer y Eros and Civilization de Herbert Marcuse. El carácter aporético de esos análisis son los que conducirán al cambio en la concepción de la crítica a partir de Habermas y, con ello, a la llamada segunda tradición de la Teoría Crítica ${ }^{27}$. En la línea existencialista destacan, por su parte, las contribuciones de Jean Paul Sartre ${ }^{28}$ y las consideraciones acerca de la alienación en Jean Hippolyte ${ }^{29}$.

En esa inflación en el uso del concepto también pudieron influir las críticas al régimen soviético que, a partir de la muerte de Stalin, se hicieron cada vez más fuertes. Así, al evaluar la situación desde las lentes de la alienación, saltaba a la vista que, en las sociedades no capitalistas, muy por el contrario de lo que una visión ingenua de la revolución podría suponer, la alienación no habia sido abolida, sino que incluso podría haberse incrementado ${ }^{30}$. El resultado general de este boom es la pérdida de circunscripción, precisión e incluso, tal y como afirma Musto, la "ambigüedad del concepto"31.

Ahora bien, si se acepta (en línea con las posiciones de los más nuevos filósofos de la alienación) que la filosofía dejó de hablar de alienación luego del

llamada "contracultura" de la década de los 60 y quedó capturada muy especialmente en el arte. Ejemplos tan destacados que hoy se consideran clásicos o de culto los encontramos en el cine (Persona (1966) de Ingmar Bergman; Un hombre que duerme (1967) de Georges Perec, adaptada cinematográficamente en 1974).

${ }^{25}$ Cf. Fromm, E., The Sane Society, Nueva York: Fawcett, 1965;Fromm, E., Marx y su concepto del hombre

${ }^{26}$ Cf. Musto, M. (ed.), De regreso a Marx: nuevas lecturas y vigencias en el mundo actual, p. 187-190.

27 Madureira, M., "La Teoría Crítica de la Escuela de Frankfurt, de la primera a la tercera generación: un recorrido histórico-sistemático", en: Revista Internacional de Filosofía Politica, 34 (2009), p. 195.

28 Sartre, J.P., La náusea, México D.F.: Época, 2005.

29 Hyppolite, J., Studies on Marx and Hegel, Nueva York: Basic Books, 1969.

${ }^{30}$ Un análisis relativo a esto lo desarrolla Golubović, Z., "New Forms of Alienation Under Real Socialism", en: Schweitzer, D. y F. Geyer (eds.), Alienation Theories and De-Alienation Strategies, Northwood: Science Reviews, 1989, pp. 95-121

${ }^{31}$ Musto, M., De regreso a Marx: nuevas lecturas y vigencias en el mundo actual, p. 188. 
boom y hasta el 1994 (año en que Schacht publica The Future of Alienation), entonces tendría que establecerse una tercera etapa en la discusión filosófica sobre la alienación, precedida por un silencio acaecido durante la década de los ochenta. Este trabajo propone que ese segundo silencio no fue tal y que no es cierto que la motivación de la discusión a partir de 1994 sea disímil a como había sido en los ochenta.

Para sustentar esto, se hará foco en la situación en torno a la discusión filosófica de la alienación durante los ochenta e inicio de los noventa, en relación con dos cuestiones:

(a) La revisión conceptual a la que somete el marxismo analítico a la teoría de Marx en los ochenta. Una de las discusiones a las que el marxismo analítico no deja de volver es la discusión sobre la alienación. Y este punto es relevante, porque la revisión que realiza el marxismo analítico de la tesis de la alienación de Marx no es meramente descriptiva: antes bien, se evalúan sus puntos altos y bajos en relación con una psicología de la acción humana.

(b) La apropiación de la discusión sobre la alienación por parte de la sociología. Lo que reviste interés a los efectos de falsear la tesis del abandono de la discusión filosófica en torno a la alienación no es que la sociologia haya desarrollado escalas para medir la alienación, i.e., que traduzca el término al lenguaje de las ciencias sociales; más bien que bajo su institucionalidad (centros y publicaciones especializadas) la sociología aglutinó no solo a los cientistas sociales, sino también a filósofos que continuaron discutiendo acerca de la alienación.

\section{Alienación en el marxismo analítico}

Las contribuciones del marxismo analítico a la discusión sobre la alienación $^{32}$ merecen una mención particular. Entre estas, destacan las de Jon Elster $(1985,1986)$, cuyas reconstrucciones analíticas del pensamiento de Marx si bien no tuvieron como eje principal el tratamiento de la alienación no dejaron de pasar por él. Por el hecho de estar presente como concepto en las disquisiciones del marxismo analítico es posible falsear la tesis de que la filosofia no habló de alienación en los ochenta.

${ }^{32}$ Debo esta sugerencia a Agustin Reyes. 
De acuerdo con Elster en An Introduction to Karl Marx³, la alienación junto con la ineficiencia y la explotación cumplen un rol normativo en la teoría de Marx porque muestran aspectos fallidos del capitalismo y, como contracara, los aspectos que vuelven deseable al comunismo. Según Elster, dentro de la teoría normativa de Marx, el concepto de alienación es el más importante. Tanto es así que "Marx valoraba el comunismo, sobre todo, porque aboliría la alienación, en los distintos sentidos de este término"34.

Asimismo, según Elster la alienación es un fenómeno indeseable provocado por el capitalismo sobre los individuos y por esto la alienación en Marx es diferente a la alienación en Hegel, quien la concibe como un aspecto del Hombre. Grosso modo, en Marx la alienación se trata de una "falta de percepción de sentido" 35 especificada en tres formas:

(1) Falta de autorrealización. De acuerdo con Elster: "Marx creía que la buena vida individual debía basarse en la autorrealización activa"36. La autorrealización consiste en la autoactualización (de una potencialidad a través de su desarrollo y despliegue) y la autoexteriorización (objetivación) plena y libre de las capacidades y habilidades de los individuos, que el capitalismo impide a la inmensa mayoría de las personas. Ahora bien, de acuerdo con Elster, la plenitud (i.e., que no haya especialización en las ocupaciones, tal y como manifestaba Marx en el famoso pasaje de La ideología alemana ${ }^{37}$ ) es una característica que vuelve utópica a la autorrealización debido a que se torna "autofrustrante". La libertad de la autorrealización, por su parte, no debe interpretarse sobre la base del comunismo entendido como una sociedad de abundancia, lo que incurriría en utopismo, sino como ausencia de coerción para elegir entre líneas alternativas de autorrealización cuando alguna se ve obturada debido a restricciones materiales de la sociedad. En línea con lo que admitiría Marx, la autorrealización de

${ }^{33}$ Cf. Elster, J., Una introducción a Karl Marx, Madrid: Siglo XXI, 1992, pp. 44-62. El tratamiento de la alienación que realiza Elster es diferente. Para empezar, se trata de un tratamiento mucho más detallado. Como el objeto de traerlo a colación aquí es simplemente dar un ejemplo, se sigue el texto de 1986 (edición 1992).

${ }_{34}$ Elster, J., Una introducción a Karl Marx, p. 44.

$204 \quad{ }^{35}$ Ibid., p. 45

${ }^{36}$ Ibid., p. 46.

${ }^{37}$ Elster refiere al famoso pasaje en que Marx y Engels sostienen que "en la sociedad comunista, donde cada individuo no tiene acotado un círculo exclusivo de actividades, sino que puede desarrollar sus aptitudes en la rama que mejor le parezca, la sociedad se encarga de regular la producción general, con lo que hace cabalmente posible que yo pueda dedicarme hoy a esto y mañana a aquello, que pueda por la mañana cazar, por la tarde pescar y por la noche apacentar el ganado, y después de comer, si me place, dedicarme a criticar, sin necesidad de ser exclusivamente cazador, pescador, pastor o crítico, según los casos" (Marx, K. y F. Engels, La ideología alemana, Madrid: Akal, 2014, p. 37) 
acuerdo con Elster es superior a otros estilos de vida (verbigracia, el consumo, la amistad, la contemplación) debido a su utilidad marginal creciente y a que ella misma constituye una fuente de estima que otros nos tienen y que es relevante para la autoestima, la cual es central para la felicidad. Siendo ventajosa, Elster se pregunta por qué no es un estilo de vida elegido más frecuentemente. Si bien la alienación podría concebirse como falta de autorrealización o falta de deseo o de oportunidades para la autorrealización o una combinación de ambas (constituyendo las llamadas "preferencias adaptativas"), Marx la asocia fundamentalmente a la falta de oportunidades. Elster añade otra opción: el deseo ineficaz de autorrealización, explicado por factores tales como la "miopía", la "aversión al riesgo" y la "tentación del francotirador"38, presentes aún en "una sociedad en la que existieran oportunidades de autorrealización"39. Esos tres factores atentarian incluso contra el surgimiento de un proyecto de sociedad de mayores oportunidades.

Asimismo, Elster examina la forma en que Marx concebía el vínculo entre autorrealización y comunidad: "la autorrealización para los otros"40, i.e., la reciprocidad en el reconocimiento de las actividades y productos que objetivan la autorrealización de todos y cada uno. Sin embargo, por más loable que esta conciliación humana parezca, solo sería posible en comunidades pequeñas de vínculo cara a cara y no en las sociedades industriales realmente existentes en las que ocurre una doble despersonalización que obtura dicha posibilidad, ya sea por el tipo de producción que impide que podamos señalar como propio lo producido, ya sea porque el mercado de masas no contacta personalmente al productor y al consumidor. Pero, además, la consideración de que tenemos sentimientos comunitarios solo porque conocemos lo que producimos para la sociedad no tiene asidero en la psicología individual de acuerdo con Elster ${ }^{41}$. Finalmente, otro modo de integrar la autorrealización con los demás es conciliar la autorrealización propia con la de otros en ámbitos pequeños donde la producción es conjunta, permitiendo que esta tenga sentido (un equipo de fútbol, una orquesta, un pequeño barco pesquero, entre otros). Sin embargo, en una sociedad industrializada ocurre que por la naturaleza del trabajo industrial (o al menos por cómo este ha tendido a desarrollarse históricamente) "la línea de montaje consigue un máximo de integración con un mínimo de

\footnotetext{
${ }^{38}$ Elster, J., Una introducción a Karl Marx, p. 50.

39 Ibid., p. 50.

40 Ibid., p. 51.

41 Ibid., p. 52.
} 
autorrealización" ${ }^{2}$, contraviniendo, nuevamente, la posibilidad de integrar autorrealización de unos y otros.

(2) Falta de autonomía. Esta consiste en la segunda forma que, según Elster, adopta la alienación en Marx. La autonomía es lo opuesto a la compulsión y consiste en el control de las acciones cuando el agente está en conocimiento de sus causas (proceso de formación del deseo en el nivel subintencional) y de sus consecuencias o realizaciones (frustración o no en el nivel supraintencional). La autonomía se daría para Marx únicamente en el comunismo debido a la transparencia sobre las acciones, las causas y las consecuencias de las mismas. Dicho esto, Elster se propone revisar el tratamiento que realiza Marx tanto de un nivel como del otro. Previo a la revisión del nivel subintencional, Elster advierte que en Marx no hay una teoría psicológica, pero que sí da pistas para emprender una reflexión en ese sentido en La ideología alemana. En el capitalismo no hay control sobre los deseos, puesto que estos en gran medida tienen dos defectos: son unilaterales y compulsivos. Tal cosa ocurre con el deseo de consumo. La unilateralidad es rechazada por Marx a causa del ideal de autorrealización plena. Sin embargo, Elster había descartado la autorrealización por utópica. Asimismo, la unilateralidad no era considerada defectuosa por Marx en todos los casos: la valía de ciertas producciones artísticas (el caso de Milton y El paraíso perdido) justifica que el artista haya tenido un deseo unilateral de producir. La compulsión, por su parte, es objetable para Marx debido a la perversión que produce: el deseo compulsivo de consumo, por ejemplo, conduce en su punto culminante a la postergación compulsiva del consumo propia del avaro. Asimismo, el caso de El paraíso perdido también objeta que el deseo compulsivo sea defectuoso siempre. Que la compulsión sea algo inherente al deseo forjado en el capitalismo es largamente testeado por Elster. En primer lugar, el consumo en el capitalismo es en gran medida no compulsivo. Así, el consumo conspicuo, las estrategias de marketing orientadas al consumo, son las excepciones y no la regla. En segundo lugar, si bien la utilidad marginal del consumo en la mayoría de los casos es decreciente, en cambio, la desutilidad marginal de no consumir es creciente. Eso para Elster quiere decir que ni en el capitalismo ni en ningún tipo de sociedad desaparecerán los deseos compulsivos hacia muchas formas de consumo. Ahora bien, distinguir meramente entre compulsión y autonomía no captura la riqueza de las motivaciones para actuar. Estas para Elster: "Provienen de hechos bioló-

${ }^{42}$ Ibid.

ARETÉ Revista de Filosofía, v. XXXIII, 2, 2021 / e-ISSN 2223-3741 
gicos profundos relacionados con los seres humanos. No son causados por el capitalismo y tampoco desaparecerán con el comunismo, lo que no quiere decir que la profundidad de los problemas y la habilidad para manejarlos sean independientes del contexto histórico"43.

Por su parte, con relación a lo supra-intencional, Elster apunta a la relación entre falta de control y transparencia. Efectivamente, hay casos en los que la falta de control se sigue de la falta de conocimiento sobre la causalidad social y una vez transparentada esta, el control se obtiene ${ }^{44}$. Sin embargo, en casos del dilema del prisionero, del hecho de que haya conocimiento de la situación no se sigue un control sobre las acciones de los individuos. Para eso, diría Marx, hace falta una "acción concertada". Pero ello, según Elster, no resuelve los problemas. En primer lugar, las economías de mercado del signo que sean son vulnerables. En segundo lugar, la "planificación central" que proponía Marx dista de ser no problemática. Y ello se debe a que las agencias centrales de planificación son sistemas sociales complejos, sus decisiones, por tanto, no son lineales y ello explica que "tienden a socavar el plan persiguiendo sus propios intereses personales o burocráticos" ${ }^{\prime 4}$. Ahora bien, suponiendo que fuera posible que hubiera una intersección perfecta entre sus intereses y el interés común "sus esfuerzos se frustrarian ante el enorme problema de reunir la información requerida para una planificación eficiente"46. En suma, defender la alienación en términos de una autonomía cercenada por el capitalismo no se sostiene. Las dificultades en la acción no necesariamente son inherentes a la acción en el capitalismo, sino que obedecen a hechos biológicos e, incluso, a problemas relativos a cómo se coordinan las actividades complejas. Asimismo, que todo lo "malo" pueda superarse de modo simultáneo derribando el capitalismo, vuelve el planteo de Marx utópico. No obstante, según Elster, ello no quiere decir que los ideales de autorrealización y de autonomía no sean valiosos, sino que tienen que elaborarse sobre bases más realistas.

(3) Alienación como dominio del capital sobre el trabajo. Esta tercera forma de alienación se consuma cuando la dominación del capital sobre el trabajo es "real" y no meramente "formal", i.e., cuando el capitalista no solo explota al obrero en razón de ser propietario de los medios de producción, pagándole a

\footnotetext{
43 Ibid., p. 55.

44 Elster coloca el ejemplo de productores cuyas expectativas con respecto a los precios determinaba su comportamiento comercial, frustrando las propias expectativas.

45 Ibid., p. 56.

46 Ibid., p. 57.
} 
cambio de que desarrolle una producción "doméstica", sino cuando extiende su dominio al proceso productivo entero (bajo la forma de una producción fabril en la que el obrero opera como apéndice de la máquina). En esta forma de alienación ocurre la pérdida de autonomía y de satisfacción con relación al trabajo, puesto que "consume la energía del obrero y agota todos sus talentos" 47 . Para Marx, la ironía y tragedia de esto es que el capital que domina es producto del propio trabajo humano. Pero dado que como trabajador no puedo controlarlo "no necesito estar ni suponer que estoy bajo el control de ningún otro"48. A esto se suma la legitimación de la explotación en virtud de la creencia en la legitimidad de la propiedad de los medios de producción. Como corolario, la alienación como sometimiento erosiona la motivación para levantarse contra el capitalismo. Una forma de legitimación de la explotación es a través de las ilusiones que la economía capitalista genera sobre sí misma y extiende a todos los agentes económicos. Esas ilusiones son denominadas "fetichismos" por Marx. A grandes rasgos, el fetichismo consiste en ocultar la estructura relacional que subyace a los atributos económicos. De los tres fetichismos enunciados por Marx (el de la mercancía, el del dinero y el del capital), Elster considera "poco convincente" al primero y significativos a los dos últimos. El fetichismo del dinero consiste en la ilusión de creer que el dinero es intrínsecamente productivo y eso sigue estando vigente (un ejemplo de ello es cuando los sindicatos abogan por incrementar los salarios nominales con independencia del poder adquisitivo). El segundo, consiste en creer que el capital es productivo por sí mismo y no a causa del trabajo. Esta versión también está vigente, de acuerdo a Elster.

Así, Elster concluye que, de las tres formas de alienación diferenciadas en el planteamiento de Marx (como falta de autorrealización, como negación de la autonomía y como sometimiento), la primera y la segunda son utópicas debido a que no son suficientemente sensibles para capturar cómo de hecho actuamos los seres humanos. En cambio, la alienación entendida como sometimiento y acrecentada por el fetichismo es aún atendible, de acuerdo a su texto de 1986.

Considerando el texto en el marco del contexto que se venía discutiendo, lo valioso de lo manifestado por Elster consiste en que da cuenta de la alienación según Marx en un tono no complaciente, desarrollando el ejercicio de podar aquellos aspectos que se revelan bien como insostenibles, bien como insuficientes, y propone profundizar en forma realista en aquellos que aún tendrían vigencia. Otro rasgo de interés a los efectos de lo que este trabajo busca

47 Ibid., p. 58.

48 Ibid., p. 59.

ARETÉ Revista de Filosofía, v. XXXIII, 2, 2021 / e-ISSN 2223-3741 
sostener se encuentra en la crítica que Elster realiza a los "neomarxistas de la Escuela de Fráncfort", quienes -en palabras de Elster- han sostenido que: "El peor aspecto del capitalismo es que los individuos no saben siquiera que están alienados. Cuando se entregan pasivamente al consumo de masas en lugar de luchar activamente por la autorrealización individual, no es porque carezcan de oportunidades para ella; es porque no tienen el deseo de hacerlo. Esta opinión paternalista, elitista y pesimista no era sostenida por Marx; al menos... en sus escritos económicos de madurez"49.

Lo que se muestra con esto es que tematizar a la alienación, no para hablar de ella en idénticos términos a la tradición, i.e., dándola por sentada, exacerbándola o desechándola, sino para reinterpretarla en términos contemporáneos, no tuvo que esperar hasta la década de los noventa: fue hecho por Elster diez años antes. Una de las motivaciones de la revisión del pensamiento de Marx por parte Elster, que consiste en criticar una de las formas que adoptó el uso del concepto de alienación ("paternalista, elitista y pesimista") no tuvo que esperar a los diagnósticos posteriores de Schacht y Jaeggi. Solo este contraejemplo debería servir de muestra de que la filosofia no desechó de su léxico el término "alienación", i.e., no hizo silencio durante los ochenta con respecto a la alienación y que el uso que se hizo del mismo antes de los noventa era no problemático.

Si aún no convence este argumento, se dará otro. Pero para este segundo argumento habrá que salirse momentáneamente del terreno de la filosofia y desplazarse hacia tierras sociológicas.

\section{El tratamiento de la alienación por parte de la sociología}

Mientras que el primer silencio de la filosofia con relación a la alienación se estaba produciendo, ciertos autores a los que se reconoce como padres del desarrollo metodológico de la tradición sociológica desarrollaron conceptos que hoy se asocian al concepto de alienación. En Francia, el caso de Émile Durkheim, fundamentalmente en torno al concepto de "anomia"50 y en el ámbito germánico,

\footnotetext{
49 Ibid., p. 45.

50 Durkheim desarrolla el concepto de anomia. Cf. Durkheim, E., La división del trabajo social, México D.F.: Colofón, 2007; Durkheim, E., El suicidio, Madrid: Akal, 2012.
} 
George Simmel, con sus disquisiciones acerca de la despersonalización ${ }^{51}$ y Max Weber, con su concepción de la "racionalización" y la "burocratización"52.

Sin embargo, no es hasta la mitad de los cincuenta del siglo pasado que la sociología norteamericana adopta como objeto de estudio propio a la alienación y persiste investigando sobre ella hasta el día de hoy. Dos son los hitos fundamentales que pueden establecerse en el desarrollo del estudio sociológico norteamericano de la alienación. En primer lugar, el debate suscitado en torno a la alienación en la American Sociological Review a partir de 1957 hasta los años ochenta por lo menos. En segundo lugar, la creación en 1972 del Research Committee on Alienation Theory and Research ${ }^{53}$ (Research Committee $n^{\circ} 36$ de la International Sociological Association, ISA) en funcionamiento aún. En el marco de ese comité se encuentra una serie de publicaciones de Geyer, Schweitzer y Heinz ${ }^{54}$, que no hacen más que mostrar que el interés hacia la temática de la alienación por parte de la sociología se mantuvo vigente durante toda esta etapa de presunto silencio por parte de la filosofia.

La primera adaptación empírica del concepto fue llevada a cabo por Leo Srole en $1956^{55}$ con la finalidad de medir grados y variedades de alienación. A partir de allí, surgieron otros intentos de adaptaciones empíricas. Desde una orientación empírico-positivista, la llevada a cabo por Melvin Seeman (1991) es considerada paradigmática, dado que a instancias de ella el concepto de alienación integra la nómina de los QOL concepts (concepto de calidad de vida) y figura en la Enciclopedia de Investigación en Calidad de Vida y Bienestar ${ }^{56}$. Melvin Seeman ha dedicado su vida a investigar en torno a la alienación de manera prácticamente ininterrumpida desde 1959 hasta la actualidad. Desde una perspectiva fenomenológica, por su parte, pueden mencionarse los abor-

${ }^{51}$ Simmel, G., Filosofia del dinero, Madrid: Instituto de Estudios Politicos, 1977.

${ }^{52}$ Cf. Weber, M., Economía y sociedad, Madrid: Fondo de Cultura Económica, 2002; Weber, M., La ética protestante y el espíritu del capitalismo, México D.F.: Ediciones Coyoacán, 1994.

${ }^{53}$ El RC en Alienation Theory and Research posee sitio web: https://www.isa-sociology.org/en/ research-networks/research-committees/rc36-alienation-theory-and-research.

${ }^{54}$ Cf. Schweitzer, D. y F. Geyer (eds.), Alienation Theories and De-alienation Strategies. Comparative Perspectives in Philosophy and the Social Sciences; Schweitzer, D. y F. Geyer, Theories of Alienation: Critical Perspectives in Philosophy and the Social Sciences, Leiden: Martinas Nijhoff, 1976; Geyer, F. y D. Schweitzer (eds.), Alienation: Problems of Meaning, Theory and Method, Londres: Routledge \& Kegan Paul, 1981; Geyer, F. y W. Heinz, Alienation, Society and the Individual: Continuity and Change in Theory and Research, Nueva Jersey: Transaction Publishers, 1992.

${ }^{55}$ Srole, L., "Social Integration and Certain Corollaries: An Exploratory Study", en: American Sociological Review, v. XXI, 6 (1956).

${ }^{56}$ Me estoy refiriendo a la Encyclopedia of Quality of Life and Well-Being Research (2014), editada por Alex C. Michalos para Springer Netherlands. 
dajes de Peter Berger y Stanley Pullberg $(1965)^{57}$, Maurice Natanson (1966) ${ }^{58}$, y los más recientes por parte de Margaret Vicker y Melissa Parris (2007) ${ }^{59}$.

Hasta ahora, sin embargo, no parecería tener sentido el traer a colación el caso de la sociología: ¿dónde están los filósofos aquí? Y la respuesta llega al abrir algunos de los libros de los sociólogos. Los filósofos se encuentran ni más ni menos que en las publicaciones compiladas en el marco del Research Committee on Alienation Theory and Research.

Pueden tomarse las obras comprendidas en las etapas que podrian ser relevantes (década de los ochenta e inicio de los noventa) para saber quiénes son los autores incluidos. En la obra Alienation: Problems of Meaning, Theory and Method de 1981, entre un tendal de sociólogos aparece un filósofo que contribuye con un artículo: se trata de Richard Schacht cuyo texto se intitula "Economic Alienation: With and Without Tears"60. Por su parte, en la publicación del 1989, Alienation Theories and De-alienation Strategies: Comparative Perspectives in Philosophy and the Social Sciences, al nombre de Schacht con su artículo "Social Structure, Social Alienation and Social Change" 61 se suma el de Mihailo Marković con "Marx's Critique of Alienation and its Emancipatory Consequences"62, el de Zagorka Golubović con "New Forms of Alienation Under Real Socialism"63 y el de Walter Feinberg con "Alienation and Moral Agency"64. Y en Alienation, Society and the Individual: Continuity and Change in Theory and Research de 1992 a Schacht y su "Hegel, Marx and Nietzsche, and the Future of Self-alienation" 65 se agregan Ludwig Nagl y su texto "Obsolescence of the

\footnotetext{
57 Berger, P., Pullberg, S., "Reification and the Sociological Critique of Consciousness", en: History and Theory, v. 4, n. 2 (1965).

58 Natanson, M., "Alienation and Social Role", en: Social Research, v. 33, n. 3 (1996).

59 Patlan-Perez, J., "Alienation" en: Mills, A. J. et al (eds.), Encyclopedia of Case Study Research, California: SAGE Publications, 2010, p. 17.

${ }^{60}$ Cf Geyer, F. y D. Schweitzer (eds.), Alienation: Problems of Meaning, Theory and Method, Londres: Routledge \& Kegan Paul, 1981.

${ }^{61}$ Cf Schweitzer, D. y F. Geyer (eds.), Alienation Theories and De-alienation Strategies. Comparative Perspectives in Philosophy and the Social Sciences, Northwood: Science Reviews, 1989.

62 Marković, M., "Marx's Critique of Alienation and its Emancipatory Consequences", en: Schweitzer, D. y F. Geyer (eds.), Alienation Theories and De-alienation Strategies. Comparative Perspectives in Philosophy and the Social Sciences, Northwood: Science Reviews, 1989.

${ }^{63} \mathrm{Cf}$. referencia en nota 21 .

${ }^{64}$ Feinberg, W., "Alienation and Moral Agency", en: Schweitzer, D. y F. Geyer (eds.), Alienation Theories and De-alienation Strategies. Comparative Perspectives in Philosophy and the Social Sciences, Northwood: Science Reviews, 1989.

65 Schacht, R., "Hegel, Marx and Nietzsche, and the Future of Self-alienation", en: Geyer, F. y W. Heinz (eds.), Alienation: Society and the Individual, Nueva Jersey: Transaction Publishers.
} 
Production Paradigm?"66 y Andrew Oldenquist con "Autonomy, Social Identities and Alienation"67. En suma, si bien estos compendios están en la línea y el marco de la investigación sociológica en torno a la alienación, en ellos se encuentran textos de filósofos que no dejaron de hablar de alienación en aquellos años en que los autores han considerado que la filosofia había dejado de hacerlo.

Esto trae como consecuencia que establecer un "segundo silencio" de la filosofia con relación a la alienación debe al menos ser matizado: antes de que Schacht publicara su obra de 1994, hubo filósofos que no dejaron de referirse a la alienación durante los ochenta y los primeros años de los noventa. Las referencias del marxismo analítico y de los filósofos que siguieron discutiendo a la alienación en el marco de las publicaciones sociológicas en que publicaron son muestra de ello.

De hecho, el propio Schacht es un filósofo que desde los años setenta se dedicó a la alienación. Su obra de 1994 incluye capítulos que habían aparecido en las obras compendiadas por los sociólogos y artículos publicados en American Philosophical Quarterly en 1986 ("Social Structure, Social Alienation and Social Change"68) y 1991 ("Hegel, Marx, Nietzsche and the Future of Alienation"69).

4. Un caso de tratamiento filosófico de la alienación bajo el "paraguas" de la sociología: Richard Schacht y El futuro de la alienación

Hasta que finalmente se decidiera a condensar en un único ejemplar de 1994 los artículos escritos entre el 1976 y 1991, habían pasado más de veinte años desde que Richard Schacht publicara su primer estudio sobre la alienación intitulado Alienation (1970). The Future of Alienation de 1994 es un compendio de su trabajo desarrollado en torno a la temática a lo largo de más de veinte años y constituye una "secuela" de aquella primera exploración ${ }^{70}$.

Lo interesante es cómo esta obra y este autor confirman la tesis anteriormente explicitada con relación a la historia de la discusión filosófica sobre

\footnotetext{
${ }^{66}$ Nagl, L., "Obsolescence of the Production Paradigm?", en: Geyer, F. y W. Heinz (eds.), Alienation, Society and the Individual: Continuity and Change in Theory and Research, Nueva Jersey: Transaction Publishers, 1992.

${ }^{67}$ Oldenquist, A., "Autonomy, Social Identities and Alienation", en: Geyer, F. y W. Heinz, Alienation, Society and the Individual: Continuity and Change in Theory and Research, New Jersey: Transaction Publishers, 1992.

${ }^{68}$ Cf. nota 34; Schacht, R., "Social Structure, Social Alienation and Social Change", en American Philosophical Quarterly, v. XXIII, 1, (1986).

${ }^{69}$ Cf. nota 38; Schacht, R., "Hegel, Marx and Nietzsche, and the Future of Self-alienation".

${ }^{70}$ Schacht, R., The Future of Alienation, Urbana: University of Illinois Press, 1994, p. 14. De esta obra no hay traducción, por lo que las citas son traducción mía.
} 
la alienación: salvo los dos últimos ${ }^{71}$, el resto de los artículos que conforman la obra de 1994 fueron capitulos de obras relativas a la alienación, editadas por sociólogos o en conexión con investigaciones de cariz sociológico ${ }^{72}$. De allí que Schacht no dude en agradecer a los sociólogos y manifieste que "la teoría de la alienación es inseparable de una variedad de largas formas de investigación concernientes a la vida social, los valores y el bien humano"73. Es decir, a partir de este momento, a los filósofos no les será posible restringir el estudio de la alienación a un único campo disciplinario; aunque las bases para entender el futuro de la alienación Schacht las situará en el trinomio Hegel-Marx-Nietzsche ${ }^{74}$.

The Future of Alienation inicia con la discusión acerca del estatuto epistémico del concepto de alienación: si permite o no realizar legitimamente el salto del "es" al "debe"; es decir, si puede ser tomada como una noción normativa sin perder, con ello, estatus científico. Para responder al problema, Schacht elabora dos líneas argumentales presentadas independientemente pero luego unificadas:

1. Si el hablar de alienación nos compromete o no con alguna clase de esencialismo.

2. Si el hablar de alienación nos permite realizar o no inferencias evaluativas críticas.

Para acometer con la primera línea argumental, Schacht esboza las posturas de quienes consideran a la alienación como un tipo de concepto capaz de unir la brecha "es-debe". Se trata de aquellos que conciben a la noción de alienación en analogía a la noción de patología (mental), definiéndola como un tipo de patología social contraída por la moderna sociedad occidental. El argumento de base sería el siguiente:

- $\mathrm{E}_{1}$ concepto de alienación se aplica a un estado de cosas alienado, $\mathrm{E}_{\mathrm{A}}$ (objetivamente verificable, describible).

- $\mathrm{E}_{\mathrm{A}}$ es patológico

- (patológico $\rightarrow$ malo)

- $\mathrm{E}_{\mathrm{A}}$ es malo

- (malo $\rightarrow$ no debe existir)

$\therefore \mathrm{E}_{\mathrm{A}}$ no debe existir

\footnotetext{
${ }^{71}$ Textos de 1986 y 1991 publicados en American Philosophical Quarterly.

${ }^{72}$ Cf. Schweitzer, D. y F. Geyer, Theories of Alienation: Critical Perspectives in Philosophy and the Social Sciences.

${ }^{73}$ Schacht, R., The Future of Alienation, p. 3.

${ }^{74}$ Cf. ibid., pp. 13-14.
} 
La inferencia se produce cuando caracterizamos al estado de cosas (alienado) como patológico, y añadimos que si algo es patológico es malo y si algo es malo entonces no debe existir. ¿Cómo se elimina $\mathrm{E}_{\mathrm{A}}$ ? Generando un nuevo estado de cosas $\left(\mathrm{E}_{\sim \mathrm{A}}\right)$ en que el concepto de alienación no pueda aplicarse.

De acuerdo con Schacht, aunque esta estrategia puede resultar atractiva, la propia radicalidad de la distinción "salud mental-enfermedad mental" ha sido cuestionada, puesto que no hay una norma extrasocial y universalmente válida que permita distinguir rígidamente en general entre los seres humanos que están mentalmente sanos de aquellos que están mentalmente enfermos, sino que estas clasificaciones son intra-societarias, relativas a un conjunto particular de normas o valores. De modo que, si la distinción está cuestionada, la posibilidad de cerrar la brecha entre el "es-debe" también queda interrumpida. Y lo mismo vale, entonces, para la noción de alienación. Asimismo, las inferencias normativas con base en la aplicación del término a ciertos individuos o grupos no están justificadas ${ }^{75}$.

Sin embargo, el rastreo de la historia del uso filosófico del concepto de alienación muestra que este cumplía una función fuertemente crítica y no meramente descriptiva, dado que se trataba de "algún tipo de separación, falta de identidad o desunidad que debe ser superada"76. Y la necesidad de esa superación se justificaba en la postulación de una esencia humana o bien de un verdadero carácter humano. La llamada autoalienación (self-alienation) supone, de acuerdo a Schacht, que el individuo se aliena con respecto a un sí mismo constituido por ciertas características que la vida humana debería tener. De modo que la autoalienación es lo opuesto de la autorrealización y pretende dar cuenta de la "deshumanización o humanización incompleta, ya sea frustrada o atrofiada"77. Mas no con respecto a la actual disposición, necesidades o deseos de un individuo, sino con relación al contenido contingente que el filósofo asigna al yo y la humanización ${ }^{78}$. Sin embargo, dado que no es posible apelar a una esencia humana, tampoco sería lícito hablar de autoalienación en este sentido de acuerdo con Schacht.

Ahora bien, esto, según el autor, obtura la posibilidad misma de dotar al término "alienación" de un sentido normativo o crítico, porque hacerlo requeriría postular una idea de esencia humana que oficie de modelo con respecto al cual

${ }^{75}$ Cf. ibid., p. 19.

${ }^{76}$ Schacht, R., The Future of Alienation, p. 19.

${ }^{77}$ Ibid., p. 20.

${ }^{78}$ Cf. ibid., p. 20. 
evaluar la vida de las personas en consideración. Lo que está implícito en el planteamiento de Schacht es que toda crítica tiene un carácter trascendente. Así, la única forma de criticar algo para este autor es establecer un patrón exterior definido con respecto al cual comparar el objeto de la evaluación crítica, desconociendo que hay otras formas de crítica posibles (no trascendentes). En particular, Jaeggi propone entender el concepto de alienación como crítico en un sentido inmanente. Aunque este modo de concebir a la crítica no esté exento de dificultades ${ }^{79}$, es un modo de crítica que no requiere presuponer forma alguna de esencialismo.

Por otra parte, Schacht admite que aún podría ser discutible la utilidad de un uso no normativo del concepto de alienación. Para acabar con esa sospecha, el autor expone la caracterización del concepto de alienación que utilizan de manera fructífera los científicos sociales. Se trata de una caracterización de la alienación que no apela a ninguna norma extrasocial ni extra-experiencial, i.e., no es esencialista, y cuya aplicación no genera consecuencias normativas, porque no considera a la alienación como algo indeseable que deba solucionarse ${ }^{80}$. De acuerdo con esta caracterización científica, la alienación es "un asunto de existencia de una o muchas variedades de discordancia (discord)" 81 clasificables en dos grupos conceptualmente diferentes, aunque extensionalmente puedan solaparse: tipos de insatisfacción (dissatisfaction) o de disfunción (dysfunction).

El grupo de discordancias entendidas como insatisfacciones son aquellas que involucran estados psicológicos (percepciones, sentimientos) y actitudes que manifiestan los individuos a las situaciones en que se encuentran y por eso Schacht las denomina instancias de S-Alienación (alienación subjetiva).

Por su parte, el grupo de discordancias entendidas como disfunciones se localizan en las relaciones sociales, i.e., en la falta de ajuste de las conductas de los individuos con respecto a las convenciones o expectativas, normativas o instituciones sociales de las que forman parte. Es por ello que Schacht las concibe como instancias de O-Alienación (alienación objetiva).

Schacht había advertido que extensionalmente las discordancias no necesariamente divergen. Así, los tres ejemplos de discordancias que el autor ofrece pueden conceptualizarse en términos subjetivos, qua insatisfacciones

\footnotetext{
${ }^{79}$ Pereira examina las limitaciones de la crítica inmanente de Jaeggi cuando se aplica a la crítica de la ideología. $C f$. Pereira, G., El asedio a la imaginación, Granada: Comares, 2018, pp. 147-148.

${ }^{80}$ Cf. Schacht, R., The Future of Alienation, p. 22.

${ }^{81}$ Ibid., p. 20.
} 
(S-Alienación) o en términos objetivos, qua disfunciones (O-Alienación), tal y como lo ilustra la tabla que sigue ${ }^{82}$ :

\begin{tabular}{|l|l|l|l|}
\hline Discordancia & \multicolumn{1}{|c|}{ Insatisfacción } & \multicolumn{1}{|c|}{ Disfunción } & $\begin{array}{l}\text { Alienación } \\
\text { resultante }\end{array}$ \\
\hline Laboral & $\begin{array}{l}\text { El trabajo me } \\
\text { disgusta" }\end{array}$ & $\begin{array}{l}\text { Ausentismo } \\
\text { Disminución de la calificación } \\
\text { de la mano de obra. }\end{array}$ & $\begin{array}{l}\text { Alienación } \\
\text { laboral }\end{array}$ \\
\hline Social & $\begin{array}{l}\text { "Me siento aislado } \\
\text { en mi comunidad" }\end{array}$ & $\begin{array}{l}\text { Alta criminalidad } \\
\text { Baja participación comunitaria }\end{array}$ & $\begin{array}{l}\text { Alienación } \\
\text { social }\end{array}$ \\
\hline Política & "Me siento política- \\
mente impotente" & $\begin{array}{l}\text { Baja participación de votantes } \\
\text { Escasa participación de } \\
\text { procesos políticos }\end{array}$ & $\begin{array}{l}\text { Alienación } \\
\text { política }\end{array}$ \\
\hline
\end{tabular}

Esta concepción científica y no esencialista tiene como resultado que la alienación es contextual ${ }^{83}$, o sea, que se establece con relación a un parámetro relativo al contexto, y que posee diferente naturaleza en las S-alienaciones y en las O-alienaciones y por ello se torna relevante la distinción entre ellas.

En el caso de las S-alienaciones, el parámetro surge de la propia perspectiva de los individuos que experimentan la insatisfacción, i.e., el "marco psicológico" integrado por factores tales como su "autocomprensión, creencias, repertorios conceptuales, actitudes, aspiraciones, deseos, sentimientos" ${ }^{4}$, marco que media entre sus percepciones de una situación (que experimentan como insatisfactoria, en relación con ese marco) y que usualmente condiciona sus reacciones a la misma. Ahora bien, dado que el marco es variable de individuo a individuo y de grupo en grupo, variarán las percepciones con relación a una misma situación social, así como la satisfacción o insatisfacción que esta genera a los involucrados. Por ello es que las S-alienaciones refieren "a la relación entre las personas constituidas psicológicamente de cierta manera y un conjunto particular de circunstancias" $\$ 5$ y, en tal sentido, seria posible mitigarlas (si fuese eso lo que uno finalmente busca, aunque la investigación

${ }^{82}$ Esta tabla es de elaboración propia y está basada en los ejemplos del autor.

${ }^{83}$ Cf. Schacht, R., The Future of Alienation, p. 26

${ }^{84}$ Ibid., p. 27.

85 Ibid. 
socio-científica no se lo plantea como objetivo) generando ajustes en las circunstancias particulares o en la "mentalidad"86 de los involucrados.

En el caso de las O-Alienaciones, por su parte, el parámetro que se toma en cuenta para establecerlas es alguna estructura social establecida. De allí que su contextualidad sea "relativa a una estructura" 87 , es decir, se trata de la disfuncionalidad de los comportamientos de los individuos con respecto a "la observancia de varias normas legales y sociales, el funcionamiento de varias instituciones, la satisfacción de diversas expectativas y prescripciones de roles" $" 88$. En ese sentido, no hay acciones disfuncionales en sí mismas, sino solamente con relación a la estructura que se tome en cuenta; i.e., la disfuncionalidad es relativa a un contexto. Variada la estructura, la acción puede no ser ya disfuncional.

En suma, la alienación tiene un carácter relacional vinculado al grado de ajuste intra-contextual entre el marco psicológico vs. la circunstancia o bien entre la estructura vs. el comportamiento efectivo. Cuando el ajuste intracontextual es incompleto, en el primer caso se trata de S-alienaciones y en el segundo caso de O-alienaciones. De acuerdo con el autor, el asunto relativo a qué hacer con esas discordancias excedería los límites de la investigación científica.

Asimismo, agrega Schacht, no es necesario que consideremos alienados a los individuos únicamente si ellos encuentran que las discordancias en sus sentimientos o sus prácticas son angustiosas. Y aunque esto parezca obvio para el caso de las O-alienaciones, no parece serlo tanto para el caso de las S-alienaciones, que dependen directamente de lo que las personas piensan y sienten ${ }^{89}$. Para ilustrarlo, Schacht ejemplifica esto con el caso de la alienación cultural, a la que define en términos de "una insatisfacción con la cultura popular"90 que experimentan aquellos que se entienden superiores a los que forman parte de ella y pretenden alejarse de dicha forma de cultura. Esta forma de alienación no está asociada a un sufrimiento, aunque esas personas están, de acuerdo a Schacht, alienadas.

Así, pues, si bien hay alienaciones que implican sufrimiento, otras no lo hacen. De modo que no es posible establecer un listado de condiciones sine

\footnotetext{
86 Ibid.

87 Ibid.

88 Ibid., p. 28.

${ }^{89}$ Cf. ibid., p. 31.

90 Ibid., p. 32.
} 
qua non de S-alienaciones o de O-alienaciones. Más bien, se trata de conceptos cluster, aplicables a instancias que comparten cierto aire de familia entre sí. Y por ello, de acuerdo a Schacht, tampoco habría algo así como "la alienación" en sentido de un macro-concepto multidimensional que permita aglomerar todas las concepciones científicas de la alienación disponibles. Lo que sí hay, en cambio, son varias alienaciones que a veces se vinculan entre sí y otras tantas veces no. Hay alienaciones que han dejado de existir, otras que siguen existiendo y otras que aún no existen. Y todas ellas son pasibles de una descripción científica que permite refinar tanto la caracterización de ciertos fenómenos como la noción misma. Pero la filosofia parecería tener bastante poco que decir acerca de la alienación.

\section{A modo de sintesis}

Para concluir, puede decirse que la historia de la discusión sobre la alienación en filosofia comprende dos etapas: la primera va de Rousseau a la bifurcación Marx/Kierkegaard, bifurcación que da cuenta de dos tradiciones en el tratamiento de la alienación. A partir de allí, el primer silencio, cuya duración aproximada es de setenta años. La segunda etapa se inicia en la década de los veinte del siglo pasado. Su comienzo es variable de acuerdo a si se toma como dato la vertiente existencialista o la marxista. Esta segunda etapa, como corolario de lo que se discutió más arriba, no ha culminado: las obras filosóficas sobre la alienación a partir de los noventa no constituyen un "renacer" del interés de la filosofia en torno a la alienación; ese interés no se interrumpió. Tampoco constituyen un modo inédito de trabajar filosóficamente la alienación. Ese quiebre, en todo caso, como consecuencia del abuso del término en los sesenta y setenta, ya se había producido antes de los noventa y del cambio de siglo. Los señalamientos críticos de Elster al tratamiento de la alienación por parte de Marx, su sospecha hacia la "concepción elitista, paternalista y pesimista" de la alienación imputable a algunos de los integrantes de la primera generación de la Escuela de Fráncfort; la adopción de la alienación como objeto de estudio de la sociología norteamericana, y en ese marco, las investigaciones filosóficas sobre la alienación, en particular el caso de Schacht, es lo que conduce a concluir que el trabajo de la filosofia en torno a la alienación siguió desarrollándose, sin pausas, entre los ochenta y los noventa.

De allí que las exposiciones filosóficas más recientes del concepto -cada una a su manera- sean herederas y ejecutoras de ese replanteamiento de los 
términos de la discusión filosófica sobre la alienación, pero de ningún modo sus autoras intelectuales. La insatisfacción con el modo como la tradición y los autores precedentes habian tratado la alienación ya había sido enunciada. Sobre esas bases ya tendidas, los autores contemporáneos erigen las investigaciones de los últimos veinticinco años sobre la alienación.

Recibido: 03/03/2021

Aceptado: 28/06/2021

\section{Bibliografia}

Althusser, L., La revolución teórica de Marx, México D.F.: Siglo XXI, 1964.

Bell, D., "Dos vías desde Marx: los temas de la alienación y la explotación, y el control de los obreros en el pensamiento socialista", en: Bell, D. (ed.), El fin de las ideologías. Sobre el agotamiento de las ideas politicas en los años cincuenta, Madrid: Centro de Publicaciones Ministerio de Trabajo y Seguridad Social, 1992, pp. 393-437.

Berardi, F., The Soul at Work. From Alienation to Autonomy, Los Ángeles: Semiotext(e), 2009.

Berger, P. y S. Pullberg, "Reification and the Sociological Critique of Consciousness", en: History and Theory, v. IV, 2, 1965, pp. 196-211. https://doi.org/10.2307/2504151

Debord, G., La sociedad del espectáculo, Rosario: Último Recurso, 2007.

Durkheim, E., La división del trabajo social, México D.F.: Colofón, 2007.

Durkheim, E., El suicidio, Madrid: Akal, 2012.

Elster, J., Una introducción a Karl Marx, Madrid: Siglo XXI, 1992.

Elster, J., Making Sense of Marx, Nueva York: Cambridge University Press, 1985.

Feinberg, W., "Alienation and Moral Agency", en: Schweitzer, D. y F. Geyer (eds.), Alienation Theories and De-alienation Strategies. Comparative Perspectives in Philosophy and the Social Sciences, Northwood: Science Reviews, 1989, pp. 197-219.

Fló, J. y M. Sambarino, Alcance y formas de la alienación, Montevideo: Ediciones de la Facultad de Humanidades y Ciencias de la Educación, 2019.

Fromm, E., Marx y su concepto del hombre, México D.F.: Fondo de Cultura Económica, 1964.

Fromm, E., The Sane Society, Nueva York: Fawcett, 1965.

Geyer, F. y D. Schweitzer (eds.), Alienation: Problems of Meaning, Theory and Method, Londres: Routledge \& Kegan Paul, 1981.

Geyer, F. y W. Heinz, Alienation, Society and the Individual: Continuity and Change in Theory and Research, Nueva Jersey: Transaction Publishers, 1992.

Geyer, F., Alienation, Ethnicity and Posmodernism, Westport: Praeger Publishers, 1996.

Golubović, Z., "New Forms of Alienation Under Real Socialism", en: Schweitzer, D. y F., Geyer (eds.), Alienation Theories and De-Alienation Strategies, Northwood: Science Reviews, 1989, pp. 95-121. 
Honneth, A., "Foreword" en: Jaeggi, R., Alienation, Nueva York: Columbia University Press, 2016.

Horkheimer, M. y T. Adorno, Dialéctica del iluminismo, Buenos Aires: Sudamericana, 1944.

Hyppolite, J., Studies on Marx and Hegel, Nueva York: Basic Books, 1969.

Jaeggi, R., Alienation, Nueva York: Columbia University Press, 2016.

Khan, N., Development of the Concept and Theory of Alienation in Marx's Writings March 1843 to August 1844. Oslo: Solum, 1995

Kierkegaard, S., La enfermedad mortal, Madrid: Trotta, 2008.

Lefebvre, H., Le matérialisme dialectique, París: Quadrige, 1990. https://doi.org/10.3917/ puf.lefeb.1990.01

Lukács, G., Historia y conciencia de clase, México D.F.: Grijalbo, 1969.

Madureira, M., "La Teoría Crítica de la Escuela de Frankfurt, de la primera a la tercera generación: un recorrido histórico-sistemático", en: Revista Internacional de Filosofia Política, 34, 2009, pp. 193-211.

Marcuse, H., Eros y civilización, Buenos Aires: Ariel, 1985.

Marković, M., "Marx's Critique of Alienation and its Emancipatory Consequences", en: Schweitzer, D. y F. Geyer (eds.), Alienation Theories and De-alienation Strategies. Comparative Perspectives in Philosophy and the Social Sciences, Northwood: Science Reviews, 1989, pp. 57-77.

Martineau, J., Time, Capitalism and Alienation. A Socio-Historical Inquiry into the Making of Modern Time, Boston: Brill, 2015.

Marx, K. y F. Engels, La ideología alemana, Madrid: Akal, 2014.

Marx, K., Manuscritos Económicos y Filosóficos, Madrid: Alianza Editorial, 2007.

Michalos, A (ed.), Encyclopedia of Quality of Life and Well-Being Research, Dordrecht: Springer Netherlands, 2014. https://doi.org/10.1007/978-94-007-0753-5

Musto, M. (ed.), De regreso a Marx: nuevas lecturas y vigencias en el mundo actual, Buenos Aires: Editorial Octubre, 2015.

Nagl, L., "Obsolescence of the Production Paradigm?”, en: Geyer, F. y W. Heinz, Alienation, Society and the Individual: Continuity and Change in Theory and Research, Nueva Jersey: Transaction Publishers, 1992, pp. 17-25.

Natanson, M., "Alienation and Social Role", en: Social Research, v. XXXIII, 3, 1996, pp. 375-388.

Nietzsche, F., Así hablaba Zaratustra, Barcelona: Brontes, 2012.

Oldenquist, A., "Autonomy, Social Identities and Alienation", en: Geyer, F. y W. Heinz, Alienation, Society and the Individual: Continuity and Change in Theory and Research, Nueva Jersey: Transaction Publishers, 1992, pp. 53-60.

Patlan-Perez, J., "Alienation" en: Mills, A. J. y otros (eds.), Encyclopedia of Case Study Research, California: SAGE Publications, 2010, pp. 16-18.

Pereira, G., El asedio a la imaginación, Granada: Comares, 2018

Rosa, H., Alienation and Acceleration. Towards a Critical Theory of Late-Modern Temporality, Malmö: NSU Press, 2010.

Sartre, J.P., La náusea, México D.F.: Época, 2005. 
Schacht, R., Alienation, Londres: George Allen \& Unwin LTD, 1971.

Schacht, R., The Future of Alienation, Urbana: University of Illinois Press, 1994.

Schacht, R., "Social Structure, Social Alienation and Social Change", en: American Philosophical Quarterly, v. XXIII, 1, (enero, 1986), pp. 47-57.

Schacht, R., "Hegel, Marx, Nietzsche, and the Future of Alienation", en: American Philosophical Quarterly, v. XXVIII, 2, (abril, 1991), pp. 125-135.

Schacht, R., "Hegel, Marx and Nietzsche, and the Future of Self-alienation", en: Geyer, F. y W. Heinz (eds.), Alienation: Society and the Individual, Nueva Jersey: Transaction Publishers, 1992, pp. 1-16. https://doi.org/10.1108/eb013142

Schmitt, R., Alienation and Freedom, Cambridge: Westview Press, 2003.

Seeman, M., "Alienation and anomie", en: Robinson, J.P. y otros (eds.), Measures of personality and social psychological attitudes, v. I , San Diego: Academic Press, 1991, pp. 291-371. https://doi.org/10.1016/B978-0-12-590241-0.50011-3

Schweitzer, D. y F. Geyer, Theories of Alienation: Critical Perspectives in Philosophy and the Social Sciences, Leiden: Martinas Nijhoff, 1976.

Schweitzer, D. y F. Geyer (eds.), Alienation Theories and De-alienation Strategies. Comparative Perspectives in Philosophy and the Social Sciences, Northwood: Science Reviews, 1989.

Simmel, G., Filosofía del dinero, Madrid: Instituto de Estudios Políticos, 1977.

Srole, L., "Social Integration and Certain Corollaries: An Exploratory Study", en: American Sociological Review, v. XXI, 6, 1956, pp. 709-716. https://doi. org/ 10.2307/2088422

Weber, M., Economía y sociedad, Madrid: Fondo de Cultura Económica, 2002.

Weber, M., La ética protestante y el espiritu del capitalismo, México D.F.: Ediciones Coyoacán, 1994. 